\title{
EFEITO DA DESINFESTAÇÃO DO SOLO PELO USO DA ENERGIA SOLAR SOBRE FUNGOS MICORRÍZICOS ARBUSCULARES ${ }^{(1)}$
}

\author{
O. RANDIG (2), C. A. B. MEDEIROS ${ }^{(3)} \&$ C. A. SPERANDIO(4)
}

\begin{abstract}
RESUMO
O uso da energia solar como método de desinfestação do solo apresenta como vantagens, além do baixo custo, ausência de riscos para a saúde humana e para o meio ambiente. Seus efeitos sobre microrganismos benéficos são, entretanto, pouco conhecidos. Objetivou-se, neste estudo, avaliar dois métodos de desi nfestação do solo por meio da energia solar, quanto aos seus efeitos sobre fungos micorrízicos arbusculares (FMA). Os experimentos foram realizados na E mbrapa Clima Temperado, Pelotas (RS), avaliando-se o efeito da solarização e de um coletor solar sobre uma população nativa de fungos micorrízicos. Após 30 dias de tratamento, o potencial de inóculo de F MA, determinado pela técnica do número mais provável, foi reduzido em $93 \%$ pela solarização e em $99 \%$ pelo coletor solar. Verificou-se, ainda, que nas plantas de milho cultivadas em solo tratado durante dois dias no coletor solar, a colonização micorrízica foi, em média, inferior a 1\%, não havendo colonização após nove dias de tratamento.
\end{abstract}

Termos de indexação: coletor solar, solarização, microrganismos.

\section{SUMMARY: EFFECTS OF SOIL DISINFESTATION BY SOLAR ENERGY ON ARBUSCULAR MYCORRHIZAL FUNGI}

Besides its low cost, solar energy usein soil disinfestation presents no risks for human heal th and theenvironment. However, its effects on beneficial microorganisms arenot well known. This study evaluated the effect of a solar col lector and sol arization on an indigenous population of arbuscular mycorrhizal fungi. I noculum potential of mycorrhizae, determined by the most probabl e number method, decreased, respectively, 93 and $99 \%$ when soil was treated during 30 days by solarization and solar collector. Mycorrhizal colonization of maize plants, grown in soil treated during two days in the solar collector, was lower than $1 \%$, and absent after nine days of treatment.

Index terms: solar collector, solarization, microorganisms.

(1) Recebido para publicação em janeiro de 2001 e aprovado em junho de 2001.

(2) Engenheiro-Agrônomo, Institut National de La Recherche Agronomique - INRA. 06606 Antibes, França. E-mail: randig@antibes.inra.fr

(3) Pesquisador da Embrapa Clima Temperado, Caixa Postal 403, CEP 96001-970 Pelotas (RS).

(4) Professor do Departamento de Fitossanidade, FAEM, Universidade Federal de Pelatas - UFPel. Caixa Postal 354, CEP 96001970 Pelotas (RS). 


\section{INTRODUÇÃO}

A desinfestação do solo com produtos químicos, real izada normal mente com brometo de metila, além do risco que representa para a saúde humana, é del etéria ao meio ambiente, por ser esseagroquímico uma das substâncias responsáveis pela rar efação da camada de ozônio (BRASIL, 1997). A utilização da energia solar como método alternativo de desinfestação do solo, em substituiç̧ão aos produtos químicos, apresenta como vantagem, além do baixo custo, a segurança para a saúde humana e para o meio ambiente. No entanto, a elevação da temperatura do solo, proporcionada por essa técnica, atinge, de forma não-seletiva, a maioria dos microrganismos do solo, sejam eles patogênicos ou benéficos para as plantas.

Dentre os microrganismos benéficos, destacamse, com papel importante na nutrição mineral de plantas e, particularmente, na absorção de fósforo, os fungos micorrízi cos arbusculares (FMA) (M edeiros et al., 1994). A maioria das espécies de FMA desempenha melhor suas funções simbióticas em temperaturas próximas a $30^{\circ} \mathrm{C}$ (J anos, 1987), suportando temperaturas de até $80^{\circ} \mathrm{C}$ por curtos períodos de tempo (Tommerup \& Kidby, 1979, 1980; McGee, 1989).

Objetivou-se, neste estudo, avaliar o efeito da elevação da temperatura do solo, resultante do processo de desi nfestação pela sol arização e pelo uso do col etor solar sobre uma populaçãonativa deFMA.

\section{MATERIAL E MÉTODOS}

O trabalho constou de dois experimentos realizados na Embrapa Clima Temperado, Pelotas (RS). No primei ro experimento, comparou-se o efeito do coletor solar (Ghini \& Bettiol, 1991) e da sol arização do sol o em tabulei ros de al venaria sobre uma população de FMA, em um delineamento experimental completamente casualizado, com quatro repetições. A duração dos tratamentos de desinfestação do solo foi de 30 dias, nos meses de janeiro/fevereiro-96, sendo a testemunha mantida em câmara fria, à temperatura de $6^{\circ} \mathrm{C}$, acondicionada em sacos de polietileno preto. O solo utilizado, Glei Vermelho-Amarelo distrófico, foi proveniente deárea cultivada com hortaliças, que continha população nativa de FMA.

$\mathrm{Na}$ solarização, o sol o foi colocado em tabuleiros dealvenaria, com dimensões de 1,37 × 2,34 x 0,10 m, irrigado até próximoà capaci dade decampo ecoberto com filme de polietileno transparente, com $100 \mu \mathrm{m}$ de espessura e aditivado anti-UV. No coletor solar, os tubos foram preenchidos com solo com umidade gravimétrica em torno de $0,2 \mathrm{~kg} \mathrm{~kg}^{-1}$.
Os efeitos dos tratamentos coletor solar e solarização sobrea população deF MA nativos foram avaliados pelo método do número mais provável (NMP), adaptado por Porter (1979). Em cada tratamento, utilizaram-se 10 níveis de diluição do solo: nível 1 - solo não diluído; nível 2 - mistura 1:3 de solo do nível 1 esol o autoclavado; nível 3-mistura 1:3 de solo do nível 2 e solo autodavado, e assim sucessivamente até o nível 10 (Sieverding, 1991). Para cada nível de diluição, foram feitas cinco repetições. Utilizou-se milho como planta hospedeira, cultivado em casa de vegetação pel o período de sete semanas. Para esse processo, as raízes foram coloridas, utilizando-se o método de Phillips \& Hayman (1970).

No segundo experimento, o solo foi tratado no col etor solar por 0, 2, 4, 6, 9 e 11 dias, durante o mês de março-96, tendo sido a testemunha mantida à sombra, em temperatura ambiente. $O$ solo utilizado teve a mesma origem daquele do primeiro experimento, com umidade gravimétrica em torno de $0,31 \mathrm{~kg} \mathrm{~kg}^{-1}$. O delineamento experimental foi o completamente casualizado, com quatro repetições.

A pós cada período de exposição, foram retirados, por repetição, aproximadamente $500 \mathrm{~g}$ de solo do coletor solar e da testemunha e distribuídos em bandejas de poliestireno expandido, preenchendose 16 células $(3,5 \times 3,5 \times 11,5 \mathrm{~cm})$ por repetição de cada tratamento. Nas bandejas, cultivou-se milho como planta indicadora da colonização micorrízica, mantendo-se uma planta por célula. O efeito do tratamento col etor solar sobre a população de FMA presente no solo foi avaliado pela determinação da percentagem de colonização micorrízica após cinco e seis semanas de cultivo, em cada um dos diferentes períodos de tratamento. A coloração das raízes foi feita conforme descrito no experimento anterior, sendo a quantificação da colonização micorrízica realizada pelo método de interseção em grade reticulada (Giovanneti \& Mosse, 1980).

Ao final dos experimentos, as plantas foram cortadas na região do colo e a parte aérea colocada para secar em estufa a $60^{\circ} \mathrm{C}$, até peso constante.

Durante o período de realização dos experimentos, foram registradas as temperaturas diárias no col etor solar e na solarização por meio de um geotermógrafo. Os val ores de temperatura do ar e insolação foram obtidos junto à estação climatológica da Embrapa Clima Temperado.

\section{RESULTADOS}

\section{Experimento coletor solar vs solarização vs testemunha}

A figura 1 mostra os dados de insolação, temperatura do ar edo solo tratado durante o período 
de realização do experimento. As temperaturas máximas obtidas no col etor solar e solarização foram de 73,0 e $52,0^{\circ} \mathrm{C}$, respectivamente, ambas no dia $7 / 02$, quando se registraram el evada temperatura do $\operatorname{ar}\left(31,2^{\circ} \mathrm{C}\right)$ e período longo de insol ação (12:14 h).

O potencial de inóculo foi significativamente diminuído após 30 dias de tratamento (Quadro 1). A solarização em tabuleiro e o coletor solar reduziram, respectivamente, em 96,3 e $99,6 \%$ o potencial de inóculo, quando comparados à testemunha.

Quadro 1. Potencial de inóculo de fungos micorrízicos arbusculares determinado pela técnica do número mais provável, em solo desinfestado por meio de um coletor solar e da solarização, por um período de 30 dias

\begin{tabular}{lc} 
Tratamento & $\begin{array}{c}\text { Propágulos infectivos/100 g } \\
\text { de solo seco }\end{array}$ \\
\hline Testemunha & $110,8 \mathrm{a}$ \\
Solarização & $4,1 \mathrm{~b}$ \\
Coletor & $0,4 \mathrm{~b}$
\end{tabular}

Médias seguidas de mesma letra não diferem entre si pelo teste de Duncan $(P>0,05)$.

\section{Experimento sobre diferentes períodos de desinfestação do solo em coletor solar}

A figura 2 mostra os dados de insolação, temperatura do ar e do solo tratado durante o período de experimentação. A temperatura máxima obtida no coletor solar foi de $68,7^{\circ} \mathrm{C}$, no dia $12 / 03 / 96$.

O coletor solar reduziu significativamente a colonização das raízes de milho por FMA, a partir do segundo dia de tratamento (Quadro 2), e, após nove dias, o decréscimo na col onização foi de $100 \%$. Não se observaram diferenças significativas na col onização micorrízica entreas médias dos períodos de cinco e seis semanas de cultivo.

As plantas cultivadas no solo desinfestado apresentaram maior produção de matéria seca em relação à testemunha (Quadro 3).

\section{DISCUSSÃO}

No primeiro experimento, as temperaturas obtidas tanto no coletor solar como na solarização inviabilizaram grande parte dos propágulos infectivos de FMA presentes no solo, reduzindo a micorrização das raízes.

As expressivas diferenças normalmente observadas entre as temperaturas máximas registradas no coletor solar e na solarização, por

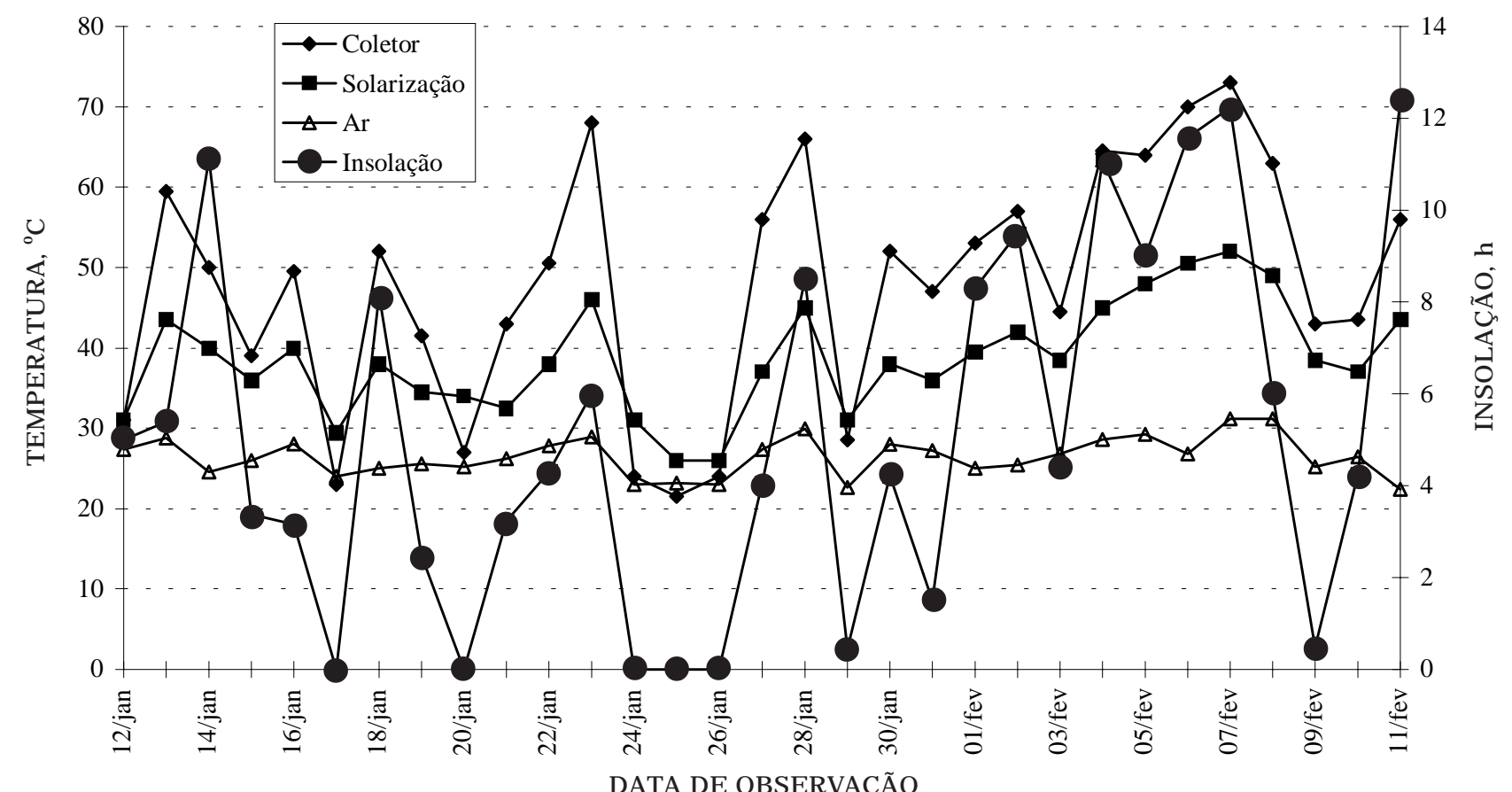

Figura 1. Temperaturas máximas do ar e do solo no coletor solar e em solarização e horas diárias de insolação no período de 12 de janeiro a 11 de fevereiro de 1996, Pelotas (RS). 


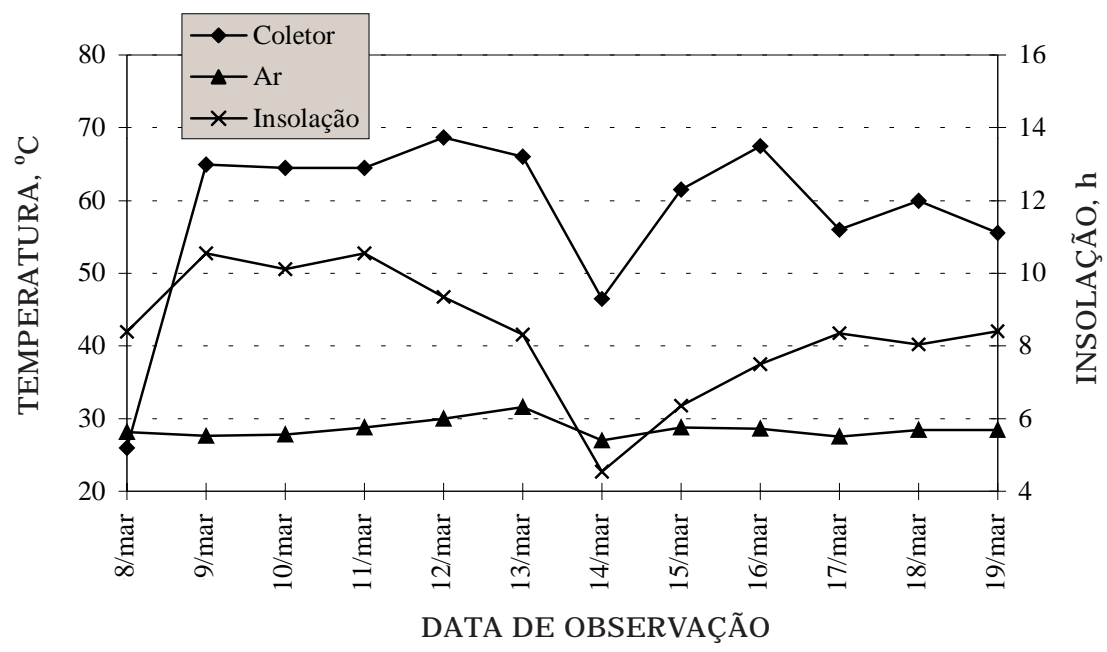

Figura 2. Temperaturas máximas do ar e do solo no coletor solar e horas diárias de insolação no período de 8 a 19 de março de 1996.

Quadro 2. Colonização de plantas de mil ho por fungos micorrízi cos arbusculares após cinco e seis semanas de cultivo, em solo desinfestado ou não (testemunha) em um coletor solar, por diferentes períodos

\begin{tabular}{|c|c|c|c|c|}
\hline \multirow{3}{*}{ Período de tratamento } & \multicolumn{4}{|c|}{ Colonização micorrízica - período de cultivo } \\
\hline & \multicolumn{2}{|c|}{ Cinco semanas } & \multicolumn{2}{|c|}{ Seis semanas } \\
\hline & Coletor & Testemunha & Coletor & Testemunha \\
\hline dias & 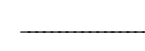 & $x_{2}$ & 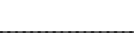 & - \\
\hline 0 & $23,02 \mathrm{a}$ & 24,17 a & $22,57 \mathrm{a}$ & 24,42 a \\
\hline 2 & $0,44 \mathrm{~b}$ & $19,60 \mathrm{a}$ & $1,50 \mathrm{~b}$ & $23,84 \mathrm{a}$ \\
\hline 4 & $0,00 \mathrm{~b}$ & $22,03 \mathrm{a}$ & $0,08 \mathrm{~b}$ & $24,24 \mathrm{a}$ \\
\hline 6 & $0,07 \mathrm{~b}$ & 28,19 a & $0,08 \mathrm{~b}$ & 30,57 a \\
\hline 9 & $0,00 \mathrm{~b}$ & $23,68 \mathrm{a}$ & $0,00 \mathrm{~b}$ & 27,95 a \\
\hline 11 & $0,00 \mathrm{~b}$ & $30,40 a$ & $0,00 \mathrm{~b}$ & $27,91 \mathrm{a}$ \\
\hline
\end{tabular}

Médias seguidas de mesma letra, na coluna, não diferem entre si pelo teste de Duncan $(P>0,05)$.

Quadro 3. Produção de matéria seca da parte aérea (MSPA) de plantas de mi lho após cinco e seis semanas de cultivo, em solo desinfestado ou não (testemunha) em um coletor solar, por diferentes períodos

\begin{tabular}{|c|c|c|c|c|}
\hline \multirow{3}{*}{ Período de tratamento } & \multicolumn{4}{|c|}{ MSPA - período de cultivo } \\
\hline & \multicolumn{2}{|c|}{ Cinco semanas } & \multicolumn{2}{|c|}{ Seis semanas } \\
\hline & Coletor & Testemunha & Coletor & Testemunha \\
\hline dias & & 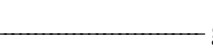 & 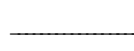 & - \\
\hline 0 & $0,32 a$ & $0,34 \mathrm{a}$ & $0,46 a$ & $0,44 a$ \\
\hline 2 & $0,53 \mathrm{a}$ & $0,39 \mathrm{~b}$ & $0,75 \mathrm{a}$ & $0,52 \mathrm{~b}$ \\
\hline 4 & $0,52 \mathrm{a}$ & $0,42 \mathrm{~b}$ & $0,82 \mathrm{a}$ & $0,60 \mathrm{~b}$ \\
\hline 6 & $0,63 a$ & $0,43 \mathrm{~b}$ & $0,67 \mathrm{a}$ & $0,44 \mathrm{~b}$ \\
\hline 9 & $0,86 a$ & $0,34 \mathrm{~b}$ & $0,68 a$ & $0,33 \mathrm{~b}$ \\
\hline 11 & $0,84 a$ & $0,39 \mathrm{~b}$ & $0,61 \mathrm{a}$ & $0,37 \mathrm{~b}$ \\
\hline Média & 0,62 & 0,39 & 0,67 & 0,45 \\
\hline
\end{tabular}

Médias seguidas de mesma letra, na linha, dentro de cada período de cultivo, não diferem entre si pelo teste de Duncan $(\mathrm{P}>0,05)$. 
exemplo, o diferencial de $22^{\circ} \mathrm{C}$ em $23 / 01$ e de $21^{\circ} \mathrm{C}$ em 7/02/96, não se traduziram em diferenças significativas na redução do potencial de inóculo, para o que, provavelmente, contribuíram, os baixos potenciais resultantes dos tratamentos. Entretanto, em relação à testemunha, o efeito do col etor solar e o da solarizaçãoforam significativos. As temperaturas máximas em torno de $52^{\circ} \mathrm{C}$, obtidas com a solarização, foram suficientemente altas para reduzir, drasticamente, o potencial de inóculo. Temperaturas mais el evadas, como as obtidas no col etor solar (73ㄷ), tiveram eficiência semelhante, sem eliminar totalmente o potencial de col onização dos fungos presentes.

A expectativa de que a maior amplitude térmica observada no coletor solar durante o período de tratamento (média em torno de $33^{\circ} \mathrm{C}$ ), quando comparada à ocorrida na solarização (média em torno de $13^{\circ} \mathrm{C}$ ), produzisse maior efeito sobre os propágulos infectivos não foi confirmada, visto que ambos os tratamentos foram altamente eficientes na redução do potencial de inóculo.

Outro aspecto a ser considerado, ao avaliar a ausência de diferenças entre os dois tratamentos, mesmo com as temperaturas mais el evadas obtidas no col etor solar, é o teor de água no solo. No coletor solar, o solo tratado possuía umidade em torno de $0,2 \mathrm{~kg} \mathrm{~kg}^{-1}$, tendo sido, na solarização, essa umi dade aumentada, quando do início do tratamento, para próximo à capacidade de campo. A baixa umidade do solo no col etor solar pode ter contribuído para a sobrevivência dos propágulos, principalmente dos esporos, mesmo nas condições de elevadas temperaturas, uma vez que a umidade elevada mel hora a condução de cal or, afetando a resistência das estruturas de repouso (Tommerup \& Kidby, 1980; Tommerup, 1983a,b).

No segundo experimento, comparando períodos de cultivo do mil ho com diferentes durações (cinco e seis semanas), procurou-se identificar possíveis diferenças na percentagem de colonização devidas a infecções secundárias, que, normal mente, ocorrem à medida que a colonização evolui. A existência de infecção secundária aumenta sobremaneira a percentagem de colonização das raízes, mascarando possíveis diferenças decorrentes dos diferentes períodos detratamento no col etor solar. Entretanto, a análise da variância não identificou diferenças significativas em relação à col onização micorrízica entre os diferentes períodos de cultivo. Souza et al. (1991) observaram indícios de infecção secundária em raízes de sorgo após 40 dias de cultivo. No entanto, o período no qual a infecção secundária iniciou-se foi variável, dependendo de condições ambientais, espécie de FMA e hospedeiro (H etrick, 1984; Bowen, 1987). No presente estudo, é provável que a infecção secundária não tenha ocorrido até a sexta semana decultivo, ou tenha ocorrido em baixos níveis, insuficientes para determinar diferenças de col onização entre os dois períodos.
Os níveis de colonização micorrízica obtidos (20-30\%) seguiram o padrão observado em al gumas espécies, dependendo do tempo de cultivo (Sanders et al., 1977). De acordo com Menge (1984), os níveis máximos de colonização seriam atingidos somente entre 10 e 14 semanas após a inoculação.

Observou-se que dois dias de tratamento do solo no col etor foram suficientes para promover redução superior a 90\% na colonização mi corrízica, dados que corroboram os obtidos por Souza et al. (1991), segundo os quais, com três dias detratamento do sol o em col etor sol ar, diminuiu-se a col onização micorrízica em torno de $75 \%$. A pós nove dias de exposi ção ao coletor solar, nãomais seobservou col onização no sistema radicular, resultado que se contrapõe aos do experimento anterior, onde, com 30 dias detratamento no col etor solar, não se eliminou a totalidade dos propágulos infectivos. Deve-sesal ientar, entretanto, quea média das temperaturas máximas do segundo experimento foi superior à do primeiro experimento, o que deve ter contribuído para a elimi nação da col onização em um menor espaço de tempo.

Outro aspecto consi derado, que pode explicar, em parte, a redução da col onização pelo coletor solar em menor tempo que no experimento anterior, referese ao teor de água do solo. A umidade gravimétrica do sol o tratado neste experimento ficou em torno de $0,31 \mathrm{~kg} \mathrm{~kg}^{-1}$, superior aos $0,2 \mathrm{~kg} \mathrm{~kg}^{-1}$ de umidade determinados no experimento anterior, o que pode ter proporcionado efeito mai or da temperatura sobre os propágul os infectivos, principal mente os esporos, de maior resistência térmica. Os resultados obtidos por Tommerup \& Kidby (1980) e Tommerup $(1983 a, b)$ evidenciaram que a temperatura teve maior efeito sobre os propágulos infectivos quando o teor de água no sol o era maior. Deve-se considerar, entretanto, que o potencial decol onização micorrízica nos dois experimentos foi estimado por métodos diferentes.

O decréscimo da população viável de FMA, a partir do segundo dia de tratamento do solo no coletor solar, não resultou em menor produção de matéria seca, em comparação às testemunhas, como seria esperado. Pel o contrário, a produção de matéria seca aumentou no tratamento com coletor solar, a despeito da ausência da col onização micorrízica. E sse aumento pode ser explicado pelo fato de que altas temperaturas do solo por longos períodos podem promover a solubilização de nutrientes até então indisponíveis à planta (Chen \& Katan, 1980; Katan, 1981; Kumar \& Yaduraju, 1992).

\section{CONCLUSÃO}

A energia solar usada para a desinfestação do solo, pela solarização em tabuleiros de alvenaria ou coletor solar, reduziu drasticamente o potencial de inóculo de fungos mi corrízicos arbusculares. 


\section{LITE RATURA CITADA}

BOWEN, G.D. The biology and physiology of infection and its development. In: SAFIR, G.R., ed. E cophysioloy of VA mycorrhizal plants. Boca Raton, CRC Press, 1987. p.27-57.

BRASIL. Ministério do Meio Ambiente, dos Recursos Hídricos e da Amazônia Legal. Brasil e a proteção da camada de Ozônio. Brasília, 1997. 37p.

CHEN, Y. \& KATAN, J. Effect of solar heating of soils by transparent polyethylene mulching on their chemical properties. Soil Sci., 130:271-277, 1980.

GHINI, R. \& BETTIOL, W. Coletor solar para desinfestação de substrato. Summa Phytopathol., 17:281-286, 1991.

GIOVANNETI, M.\& MOSSE, B. An evaluation of techniques for measuring vesicular-arbuscular mycorrhizal infection in roots. New Phytol., 84:489-500, 1980.

HETRICK, B.A.D. E cology of V.A. mycorrhizal fungy. In: POWELL, C.L. \& BAGYARAJ, D.J ., eds. VA Mycorrhiza. Boca Raton, CRC Press, 1984. p.35-55.

J ANOS, D.P. VA Mycorrhizas in humid tropical ecosystems. In: SAFIR, G.R., ed. Ecophysioloy of VA mycorrhizal plants. Boca Raton, CRC Press, 1987. p.107-134.

KATAN, J. Solar heating (solarization) of soil for control of soilborne pests. Ann. Rev. Phytopathol., 19:211-236, 1981.

KUMAR, B. \& YADURAJ U, N.T. Effects of solarization on the temperature and physico-chemical properties of soil. Plasticulture, 94:13-20, 1992.

MCGEE, P.A. Variation in propagule numbers of vesiculararbuscular mycorrhizal fungi in a semi-arid soil. Mycol. Res., 92:28-33, 1989.

MEDEIROS, C.A.B.; CLARK, R.B. \& ELLIS, J.R. Growth and nutrient uptake of sorghum cultivated with vesiculararbuscular mycorrhiza isolates at varying pH. Mycorrhiza, 4:185-191, 1994.
MENGE, J.A. I noculum production. In: POWELL, C.L. \& BAGYARAJ, D.J., eds. VA Mycorrhiza. Boca Raton, CRC Press, 1984. p.187-203.

PHILLIPS, J.M. \& HAYMAN, D.S. I mproved procedures for clearing roots and staining parasitic and vesiculararbuscular mycorrhizal fungi for rapid assessment of infection. Trans. Brit. Mycol. Soc., 55:158-161, 1970.

PORTER, W.M. The "Most Probable Number" method for enumerating infective propagules of vesicular-arbuscular mycorrizal fungi in soil. Aust. J. Soil Res., 17:515-519, 1979.

SANDERS, F.E.;TINKER, P.B.; BLACK, R.L.B. \& PALMERLEY, S.M. The development of endomycorrhizal root systems. I. Spread of infection and growth-promoting effects with four species of vesicular-arbuscular endophyte. New Phytol., 78:257-268, 1977.

SIEVERDING, E. Vesicular-abuscular mycorrhiza management in tropical agrosystems. Eschborn, Deutsche Gesellschaft für, 1991. 371p.

SOUZA, F.A.; VASQUEZ, G.A.; SAITO, E.S.; GHINI, R. \& GUERRA, J.G.M. Desinfestação de solo para inoculação com fungos micorrízicos vesículo- arbusculares (MVA) usando um coletor solar. In: REUNIÃO BRASILEIRA SOBRE MICORRIZAS, 4., Mendes, 1991. Programa e Resumos. Itaguaí, EMBRAPA/UFRRJ , 1991. p.192.

TOMMERUP, I.C. Spore dormancy in vesicular-arbuscular mycorrhizal fungi. Trans. Br. Mycol. Soc., 81:37-45, 1983a.

TOMMERUP, I.C. Temperature relations of spore germination and hyphal growth of vesicular-arbuscular mycorrhizal fungy in soil. Trans. Br. Mycol. Soc., 81:381-387, 1983b.

TOMMERUP, I.C. \& KIDBY, D.K. Preservation of spores of vesicular-arbuscular endophytes by L-Drying. Appl. Environ. Mycrobiol., 37:831-835, 1979.

TOMMERUP, I.C. \& KIDBY, D.K. Production of asseptic spores of vesicular-arbuscular endophytes and their viability after chemical and physical stress. Appl. Environ. Microbiol., 39:1111-1119, 1980 